\title{
How to select the best upfront therapy for metastatic disease? Focus on ALK-rearranged non-small cell lung cancer (NSCLC)
}

\author{
Bing Xia ${ }^{1 \#}$, Misako Nagasaka ${ }^{2,3 \#}$, Viola W. Zhu ${ }^{4}$, Sai-Hong Ignatius $\mathrm{Ou}^{4}$, Ross A. Soo ${ }^{5}$ \\ ${ }^{1}$ USC Norris Comprehensive Cancer Center, Keck School of Medicine of USC, Los Angeles, CA, USA; ${ }^{2}$ Karmanos Cancer Institute, Wayne \\ State University School of Medicine, Detroit, MI, USA; ${ }^{3}$ St. Marianna University Graduate School of Medicine, Kawaski, Japan; ${ }^{4}$ Chao Family \\ Comprehensive Cancer Center, Department of Medicine, Division of Hematology-Oncology, University of California Irvine School of Medicine, \\ Orange, CA, USA; ${ }^{5}$ Department of Haematology-Oncology, National University Cancer Institute of Singapore, National University Health System, \\ Singapore, Singapore \\ Contributions: (I) Conception and design: All authors; (II) Administrative support: B Xia, RA Soo; (III) Provision of study materials or patients: B Xia, \\ M Nagasaka, SHI Ou, RA Soo; (IV) Collection and assembly of data: All authors; (V) Data analysis and interpretation: All authors; (VI) Manuscript \\ writing: All authors; (VII) Final approval of manuscript: All authors. \\ \#These authors contributed equally to this work. \\ Correspondence to: Ross A. Soo. Department of Haematology-Oncology, National University Cancer Institute, National University Health System, 1E \\ Kent Ridge Road, NUHS Tower Block Level 7, Singapore 119228, Singapore. Email: ross_soo@nuhs.edu.sg.
}

\begin{abstract}
Anaplastic lymphoma kinase (ALK) inhibitors have demonstrated robust clinical activity in patients with $A L K$-rearranged lung cancers. The echinoderm microtubule-associated protein-like $(E M L)$ - $A L K$ translocation was first discovered in 2007 and 4 years later, crizotinib, a first-generation ALK inhibitor was approved. Since then, subsequent generations of ALK inhibitors have demonstrated superior efficacy and better CNS activity compared to crizotinib. Alectinib and brigatinib, both second-generation ALK inhibitors have been compared directly to crizotinib in the first-line setting and has demonstrated improved progression free survival (PFS) and intracranial response. Ceritinib, another second-generation ALK inhibitor has been shown to be superior to chemotherapy in $A L K$-rearranged disease with good CNS activity. Initial responses to ALK inhibitors are not always durable and resistance can occur as on-target or off-target alterations. Lorlatinib, a third-generation ALK inhibitor, has demonstrated activity in the treatment naïve setting and in resistance to crizotinib and second-generation ALK inhibitors. Lorlatinib has also shown improved PFS in patients harboring EML4-ALK variant 3, which is associated with the development of $A L K$ resistance mutations, specifically G1202R. Another new ALK inhibitor, ensartinib, has demonstrated efficacy in the first-line setting and in alectinib refractory disease. Additional studies are underway examining mechanisms of resistance and best treatment options post resistance.
\end{abstract}

Keywords: Non-small cell lung cancer (NSCLC); anaplastic lymphoma kinase (ALK); anaplastic lymphoma kinase tyrosine kinase inhibitor (ALK TKIs); targeted therapy

Submitted Feb 24, 2020. Accepted for publication Jun 30, 2020.

doi: 10.21037/tlcr-20-331

View this article at: http://dx.doi.org/10.21037/tlcr-20-331

\section{Introduction}

Lung cancer is the most common cause of cancer-related death in both men and women worldwide (1). About 4-6\% of non-small cell lung cancers (NSCLCs) develop an inversion on the short arm of chromosome 2 that joins the $5^{\prime}$ end of the echinoderm microtubule-associated protein-like
4 (EML4) with the 3' end of the anaplastic lymphoma kinase $(A L K)$ gene, which results in a chimeric protein $E M L 4-A L K$ $(2,3)$.

EML4-ALK fusions have distinct clinical characteristics and most commonly seen in patients with adenocarcinoma, young age, and a never/light smoking history (4).

The EML4-ALK fusion can be detected by fluorescent 


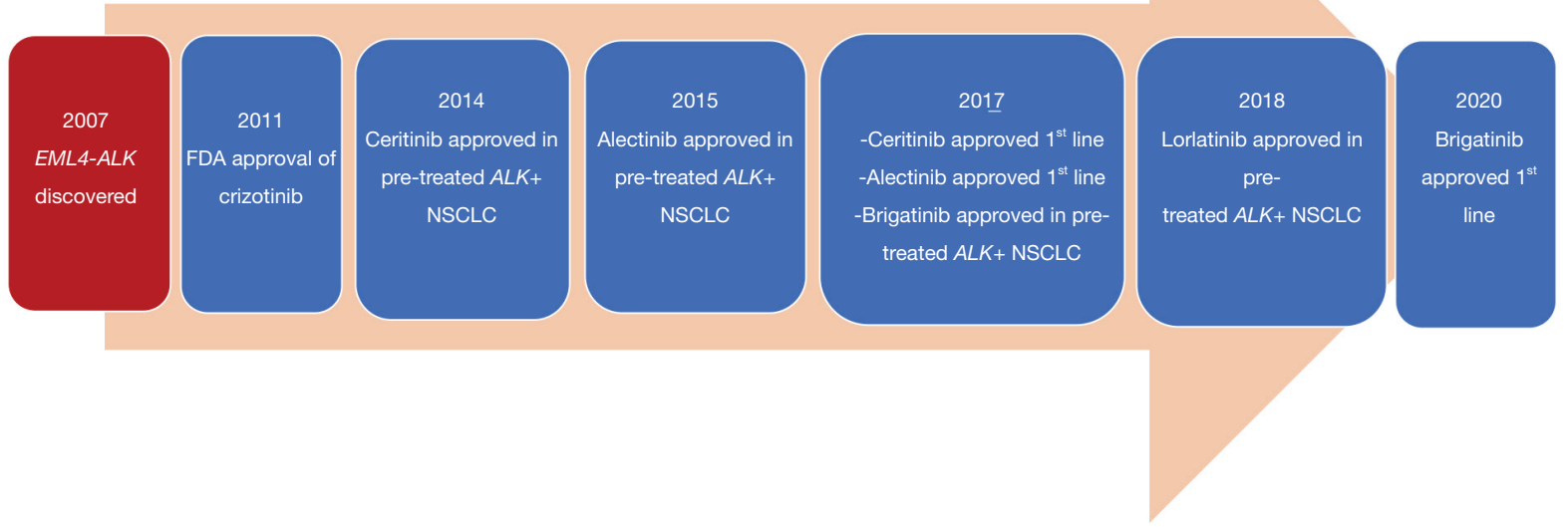

Figure 1 Timeline of FDA approvals for ALK TKIs. ALK, anaplastic lymphoma kinase; TKIs, tyrosine kinase inhibitors.

Table 1 The activity of ALK TKIs in the first-line setting in advanced $A L K$-rearranged NSCLC

\begin{tabular}{lccc}
\hline ALK TKI & Median PFS (95\% Cl) & ORR (95\% Cl) & Intracranial response (95\% Cl) \\
\hline Crizotinib (10) & $10.9(8.3-13.9)$ & $74 \%(67-81 \%)$ & NA \\
Ceritinib (11) & $16.6(12.6-27.2)$ & $72.5 \%(65.5-78.7 \%)$ & $72.7 \%(49.8-89.3 \%)$ \\
Alectinib (7,12) & $34.8(17.7-\mathrm{NR})$ & $82.9 \%(76-88.5 \%)$ & $82.9 \%(76-88.5 \%)$ \\
Brigatinib (8,13) & $29.4(21.2-\mathrm{NR})$ & $71 \%(62-78 \%)$ & $78 \%(52-94 \%)$ \\
Lorlatinib (9) & NR (11.4-NR) & $90 \%(73.5-97.9 \%)$ & $66.7 \%(9.4-99.2 \%)$ \\
Ensartinib (14) & $26.2(9.2-\mathrm{NR})$ & $80 \%(54.8-93 \%)$ & $64.3 \%(38.8-83.7 \%)$ \\
\hline
\end{tabular}

ALK, anaplastic lymphoma kinase; TKIs, tyrosine kinase inhibitors; $\mathrm{Cl}$, confidence interval; NR, not reached; ORR, overall response rate; PFS, progression free survival.

in situ hybridization (FISH), immunohistochemistry (IHC), or next generation sequencing (NGS) and are very sensitive to treatment of ALK tyrosine kinase inhibitors (TKIs). With NGS, at least 90 distinct fusion partners have been identified in $A L K$-rearranged NSCLC (5).

After the $E M L 4-A L K$ translocation was first discovered in a lung cancer cell line in 2007 , crizotinib, a firstgeneration ALK inhibitor was approved merely 4 years later. A timeline of key events in the development of firstline ALK TKIs is shown in Figure 1.

Since then, second-generation ALK inhibitor, ceritinib has been shown to be superior to chemotherapy while alectinib and brigatinib have demonstrated superior efficacy and better CNS activity compared to crizotinib (6-8). Third-generation lorlatinib has now demonstrated efficacy in the treatment naïve setting and after resistance to first and secondgeneration of ALK TKIs (9). In the US, crizotinib, alectinib, brigatinib,and ceritinib are ALK TKIs approved in the firstline. Ceritinib, brigatinib, and lorlatinib have been approved for second or in further lines of therapy. Progression free survival (PFS), response rate, and intracranial activity of crizotinib, ceritinib, alectinib, brigatinib, lorlatinib, and ensartinib is summarized in Table 1.

Comparison of median PFS among phase 3 ALK TKI trials is shown in Figure 2.

\section{Overview of ALK TKIs}

\section{Crizotinib}

Crizotinib was compared to chemotherapy in a phase 3 


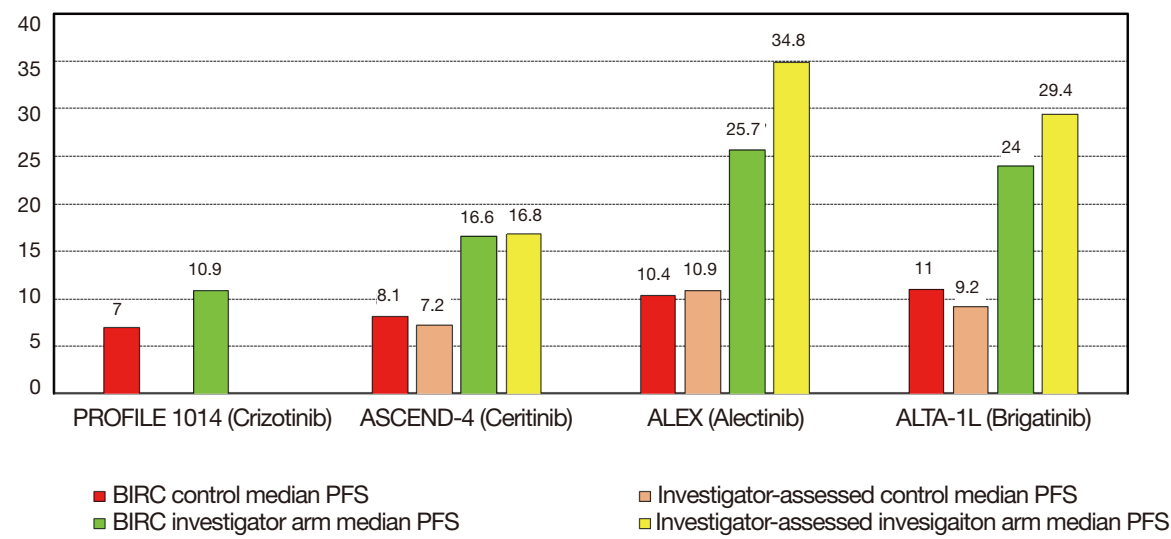

Figure 2 Comparison of median PFS among phase 3 ALK TKI trials (7,8,10-13). PFS, progression free survival; ALK, anaplastic lymphoma kinase; TKIs, tyrosine kinase inhibitors.

PROFILE 1014 trial comparing crizotinib $250 \mathrm{mg}$ twice daily to chemotherapy with carboplatin/cisplatin plus pemetrexed in patients with $A L K$-rearranged nonsquamous NSCLC. Crizotinib was found to have superior response rate and PFS compared to chemotherapy and better quality of life. The median PFS of the crizotinib group was $10.9 \mathrm{vs}$. 7.9 months in the chemotherapy arm [hazard ratio (HR) for progression or death with crizotinib, $0.45 ; 95 \%$ confidence interval (CI), 0.35-0.60; $\mathrm{P}<0.001]$. Objective response rate of crizotinib was $74 \%$ compared to $45 \%$ with chemotherapy $(\mathrm{P}<0.001)(10)$. Four-year overall survival (OS) was $56.6 \%$ for crizotinib and $49.1 \%$ for chemotherapy. HR for death with crizotinib was not significantly different than with chemotherapy, 0.760; 95\% CI, 0.548-1.052 ( $\mathrm{P}=0.0978$ ) (15). The most common toxicities of crizotinib included vision disorder (71\%), diarrhea (61\%), and edema (49\%). A quality of life assessment was performed on this study and found that patients treated with crizotinib, compared to chemotherapy, had decreased lung cancer symptoms and improved quality of life (10).

\section{Ceritinib}

The efficacy of ceritinib was shown in a phase 3 ASCEND-4 open-label randomized trial that compared oral ceritinib $750 \mathrm{mg} /$ day compared to platinum-pemetrexed chemotherapy for four cycles followed by maintenance pemetrexed. Ceritinib demonstrated superior PFS compared to chemotherapy with median PFS of 16.6 (95\% CI, 12.6-27.2) months in the ceritinib group vs. 8.1 (95\% CI, 5.8-11.1) months in the chemotherapy group. Overall response rate (ORR) in the ceritinib group was 72.5\% (95\% CI, $65.5-78.7 \%$ ) compared to $26.7 \%$ (95\% CI, $20.5-33.7 \%$ ) in the chemotherapy group. Ceritinib was demonstrated to have CNS activity with a CNS response rate of $72.7 \%$ (95\% CI, 49.8-89.3\%) in patients with measurable baseline brain metastases. The most common side effects of ceritinib were gastrointestinal related symptoms including diarrhea $(85 \%)$, nausea $(69 \%)$, and vomiting (66\%). However, the toxicity profile of ceritinib was rather significant with the dose decreases or interruptions rates to be $80 \%$ with ceritinib compared to $45 \%$ with chemotherapy (11). A later trial concluded that ceritinib at $450 \mathrm{mg}$ taken with food had similar activity to $750 \mathrm{mg}$ taken fasting and reduced gastrointestinal toxicities (16).

\section{Alectinib}

Alectinib has demonstrated efficacy in the first-line setting in the treatment of $A L K$-rearranged lung cancer in the phase III ALEX study (7). This trial compared alectinib $600 \mathrm{mg}$ twice daily with crizotinib $250 \mathrm{mg}$ twice daily in treatment naïve patients. Alectinib showed better efficacy with superior 12-month event free survival, PFS, and decreased CNS progression. The ORR was higher in the alectinib group $(82.9 \%$; $95 \%$ CI, $76.0-88.5 \%)$ compared to crizotinib (75.5\%; 95\% CI, 67.8-82.1\%) but did not reach statistical significance $(\mathrm{P}=0.09)$. The 12 -month event free survival was significantly longer with alectinib, $68.4 \%$ (95\% CI, 61.0-75.9\%) compared with crizotinib $48.7 \%$ (95\% CI, 40.4-56.9\%); HR for PFS, 0.47 (95\% CI, 0.34$0.65) ; \mathrm{P}<0.001$. CNS disease was better controlled with 
alectinib than with crizotinib. CNS progression occurred in 18 patients $(12 \%)$ in the alectinib group compared to 68 patients $(45 \%)$ in the crizotinib group (HR, 0.16; $95 \%$ CI, 0.10-0.28; $\mathrm{P}<0.001)$ (7). Updated data from the ALEX trial showed a median investigator-assessed PFS of 34.8 [95\% CI, 17.7-not reached (NR)] months in the alectinib group compared to 10.9 (95\% CI, 9.1-12.9) months with crizotinib (12). Five-year survival rate was $62.5 \%$ (95\% CI, 54.3-70.8\%) with alectinib vs. $45.5 \%$ (95\% CI, $33.6-$ $57.4 \%$ ) with crizotinib although the OS data remains (17).

The alectinib group experienced less grade 3 to 5 toxicities, $41 \%$ with alectinib versus $50 \%$ with crizotinib. Any grade adverse events were similar between the two groups with $97 \%$ of patients in both groups experiencing any adverse even. The most common side effects in the crizotinib group were gastrointestinal symptoms including nausea $(48 \%)$, diarrhea (45\%), and vomiting (38\%). Patients in the alectinib group most commonly experienced peripheral edema (17\%), anemia (20\%) and increases in liver function tests including elevations in ALT (15\%), AST (14\%), and bilirubin (15\%).

Alectinib was also studied in the J-ALEX and ALESIA trials $(18,19)$. The J-ALEX was a randomized phase III study that evaluated crizotinib versus alectinib in ALK inhibitor naïve Japanese patients. Alectinib again showed superior PFS compared to crizotinib with median PFS NR in the alectinib arm (95\% CI, 20.3-not estimated) and 10.2 months in the crizotinib arm (95\% CI, 8.2-12.0); HR, 0.34 (99.7\% CI, 0.17-0.71); $\mathrm{P}<0.0001$. Objective response rate was also higher with alectinib compared to crizotinib 92\% (95\% CI, 85.6-97.5\%) and 79\% (95\% CI, 70.5-87.3\%) respectively (18). The ALESIA trial also demonstrated improved PFS of alectinib compared to crizotinib in the Asian patients which included investigational sites in China, South Korea, and Thailand. PFS was significantly better with alectinib compared to crizotinib. Median PFS was NR in the alectinib group compared with 11.1 months in the crizotinib group (HR, 0.22; 95\% CI, 0.13-0.38; $\mathrm{P}<0.0001$ ). Again, alectinib was shown to have better objective response rate compared to crizotinib, $91 \%$ vs. $77 \%$, and longer duration of response (HR, 0.22; 95\% CI, 0.12-0.40; $\mathrm{P}<0.0001$ ). CNS response was also better with alectinib $73 \%$ compared to $22 \%$ with crizotinib (19).

The BFAST study evaluated the utility of stand-alone blood-based NGS in biomarker detection. In one arm of this study, clinical activity of alectinib was evaluated in those who had $A L K$ positivity detected by blood-based biomarker testing alone. Treatment with alectinib resulted in an investigator-confirmed ORR of $87.4 \%$ and PFS of $78.38 \%$ in those who were $A L K+$ by blood-based NGS, demonstrating the effectiveness of using blood-based NGS in biomarker detection and clinical decision making (20).

\section{Brigatinib}

A second-generation ALK inhibitor, brigatinib, was developed along with alectinib and ceritinib for patients who had progressed on crizotinib. Brigatinib, in the first-line setting, was studied in the ALTA-1L trial that randomized patients with treatment naïve $A L K$-rearranged NSCLC to brigatinib $180 \mathrm{mg}$ once daily (with $90 \mathrm{mg}$ daily for an initial 7-day lead-in period) compared to crizotinib at $250 \mathrm{mg}$ twice daily. Brigatinib was found to have superior efficacy compared to crizotinib with better PFS and intracranial response. The estimated 12-month PFS was 67\% (95\% CI, 56-75\%) with brigatinib compared with $43 \%$ with crizotinib (95\% CI, 32-53); HR for disease progression or death, 0.49 (95\% CI, 0.33-0.74); $\mathrm{P}<0.001$. The ORR was numerically higher in the brigatinib group $71 \%$ (95\% CI, 62-78\%) compared to crizotinib 60\% (95\% CI, 51-68\%), odds ratio 1.59 (95\% CI, 0.96-2.62). Intracranial response rates were significantly higher in the brigatinib group than crizotinib group at $78 \%$ (95\% CI, 52-94\%) and $29 \%(95 \%$ CI, 11-52\%), respectively, odds ratio 10.42 (95\% CI, 1.90 57.05) (8). Updated data from the ALTA-1L study showed blinded independent review committee (BIRC) PFS of 24.0 (95\% CI, 18.5-NR) months with brigatinib compared to 11.0 (95\% CI, 9.2-12.9) months with crizotinib. Investigator-assessed PFS demonstrated median PFS of 29.4 (95\% CI, 21.2-NR) months with brigatinib vs. 9.2 (95\% CI, 7.4-12.9) months with crizotinib. Intracranial PFS by BIRC was significantly better in the brigatinib group compared to crizotinib, 24 (95\% CI, 12.9-NR) months compared to 5.5 (95\% CI, 3.7-7.5) months respectively (13). However, the efficacy of brigatinib in alectinib-refractory disease demonstrated limited activity, with an ORR of $17 \%$ and median PFS of 4.4 months (21).

Adverse events were similar between the brigatinib and crizotinib groups with $97 \%$ of the brigatinib patients and $100 \%$ of the crizotinib patients experiencing any grade adverse event. The most common side effects in the brigatinib group included diarrhea (49\%), increased creatine kinase level (39\%), and nausea (26\%). The most 
common side effect of the crizotinib group included nausea $(56 \%)$, diarrhea (55\%) and vomiting (39\%) (8).

\section{Lorlatinib}

Lorlatinib is a reversible potent third-generation TKI that is highly selective and targets $A L K$ and ROS1 kinase domain. It was developed to overcome resistant $A L K$ mutants including the common G1202R. Lorlatinib has excellent penetration to the blood brain barrier and its efficacy has also been demonstrated even in patients with intracranial metastases after progression on second-generation ALK TKIs.

In a previously reported phase I portion of a phase I-II study, the ORR with lorlatinib was objective $45 \%$ (19 out of 41) and durable responses with the median duration of response of 12.4 (95\% CI, 6.5-NR) months in $A L K$-positive group. Many of these patients had received multiple lines of therapy and had intracranial metastases. Responses were seen in those previously treated with second-generation ALK TKIs and those who had only received crizotinib (22).

Lorlatinib received FDA accelerated approval on November 2, 2018 for the treatment of advanced $A L K$ rearranged NSCLC after progression on crizotinib followed by at least one other ALK TKI, or those whose disease have progressed on alectinib or ceritinib as the first ALK inhibitor (23). The approval was based on ORR, intracranial response rate and duration of response with lorlatinib in a subgroup of 215 patients in a phase II study (NCT01970865) enrolling patients who had $A L K$ rearranged NSCLC who had progressed on at least one line of ALK TKI. In this study, lorlatinib demonstrated efficacy and CNS activity in all groups including treatment naïve and prior treated patients up to three prior lines of therapy. The ORR in the $A L K$ cohort was $90 \%$ (95\% CI, $73.5-97.9 \%)$ in treatment naïve patients, $69.5 \%$ (95\% CI, $56.1-80.8 \%)$ in those who received previous crizotinib, $32.1 \%$ (95\% CI, 15.9-52.4\%) in those who received a non-crizotinib TKI, and $38.7 \%$ (95\% CI, 29.6-48.5\%) in those who received two or more ALK TKIs. The estimated median duration of response was 12.5 months.

Intracranial ORR was $60 \%$ with complete response in $21 \%$ and an estimated median duration of intracranial response of 19.5 months. Intracranial response was observed across all cohorts, including 66.7\% (95\% CI, 9.4-99.2\%) in treatment native patients, $87.0 \%$ (95\% CI, 66.2-97.2\%) in those who received previous crizotinib, $55.6 \%$ (95\% CI,
$21.2-86.3 \%)$ in those who received a non-crizotinib TKI, and $53.1 \%$ (95\% CI, 38.3-67.5\%) in those who received two or more ALK TKIs (9).

The most common adverse events observed were hypercholesterolemia (66\%), hypertriglyceridemia (45\%), and edema (41\%). Grade 4 toxicity was rare with $1 \%$ hypercholesterolemia, 3\% hypertriglyceridemia, and $<1 \%$ elevated lipase, acute respiratory failure, and elevation of blood potassium (9).

\section{Ensartinib}

Ensartinib was evaluated in a phase I/II clinical trial in ALKrearranged lung cancer patients who were treatment naïve, treated with prior ALK TKI, and brain metastases. In all $A L K$-positive patients treated with $\geq 200 \mathrm{mg}$, the response rate was $60 \%$ and median PFS was 9.2 months. In the treatment native population, the response rate was $80 \%$ and the median PFS was 26.2 months whereas the response rate was $69 \%$ and PFS was 9.0 months in patients previously treated with crizotinib alone. Intracranial response rate was $64 \%$ (14). In alectinib-refractory disease, ensartinib demonstrated a RR of $23 \%$ and a disease control rate (DCR) of $50 \%$ (24). The most common treatment related toxicities reported for ensartinib includes rash reported in $56 \%$, nausea in $36 \%$, pruritis in $28 \%$, vomiting in $26 \%$ and fatigue in $22 \%$ of patients (14). The eXalt3 trial is an ongoing randomized phase III trial (NCT02767804) that is comparing ensartinib with crizotinib in the first-line setting.

\section{Selection of ALK inhibitors}

For the most part, targeting $A L K$-rearranged NSCLC has been a therapeutic success with multiple drugs approved. However, these patients ultimately progress with ontarget (ALK-dependent) or off-target (ALK-independent) resistance mechanisms (25-28). Therefore, the most optimal sequencing of the ALK inhibitors remains to be an ongoing field of further investigation. The selection of an ALK TKI is based on factors including systemic and CNS activity of the ALK TKI, various EML4-ALK variants, mechanisms of resistance as well as the toxicity profile.

In a newly diagnosed, advanced $A L K$-rearranged NSCLC, alectinib is generally recommended as frontline therapy due to its superiority in PFS (7) and CNS activity when compared to crizotinib (29). Brigatinib has also emerged as a potential option in the frontline setting after 
demonstrating favorable responses over crizotinib in the phase III ATLA-1L trial although it has a rare but real risk of early-onset pulmonary toxicity that requires close monitoring (30).

While alectinib is generally recommended as frontline therapy in the US due to its superiority in PFS and CNS activity when compared to crizotinib $(7,29)$, crizotinib remains an option as first-line treatment in other regions of the world (31). Although some studies have shown the potential OS benefit of sequential use of a secondgeneration ALK inhibitor post crizotinib, these were retrospective evaluations (32-35). The updated analysis of ALEX suggests the OS benefit with first-line alectinib use as seen from the 5 -year survival rate of $62.5 \%$ (95\% CI, $54.3-70.8 \%$ ) with alectinib vs. $45.5 \%$ (95\% CI, 33.6-57.4\%) with crizotinib although the OS data remains immature and the median OS with alectinib was not estimable (17). Indeed, previous studies have suggested that initial use of a second-generation ALK TKI may be superior to sequential treatment with first-generation followed by secondgeneration TKIs $(7,8,11,18)$. However, in a situation where crizotinib was given as the first-line of therapy, secondgeneration ALK TKIs, for example, ceritinib, alectinib, brigatinib, and ensartinib, have all shown efficacy in those patients who have progressed on crizotinib (36).

Whether administered directly after first-line secondgeneration ALK TKI, or after sequencing with crizotonib followed by second-generation ALK TKI, the use of the third-generation ALK TKI, lorlatinib, has been FDA approved and has been generally accepted for use in this setting, although the use of stereotactic body radiation therapy (SBRT) or other local procedures such as ablation, cryotherapy or even resection maybe be considered through careful multidisciplinary discussion for oligometastatic disease progression with the continuation of the ALK inhibitors that was being used at that time $(31,37)$.

Also important to note is that platinum doublet chemotherapy is a valid treatment option for patients with $A L K$ translocation (38). While late generation ALK TKIs may be useful in targeting resistant on-target ( $A L K$ dependent) mutations in $A L K$ (for example, lorlatinib for G1202R), off-target resistance ( $A L K$-independent) may be better treated with platinum doublet chemotherapy $(31,37)$. Chemotherapy may be also considered for scenarios where multiple sites of progression is seen. In theory, in a situation where the progression of disease includes the brain, consideration should be given to utilize an ALK TKI in combination with chemotherapy. Since this strategy is supported mainly through retrospective observations (38), additional evidence from prospective clinical trials would be important to document the effects of combination as well as its safety.

In a scenario where both lorlatinib is not available and platinum doublet chemotherapy is not an option (or had already been given), there are data to support the use of some second-generation ALK inhibitors after failing a second-generation ALK TKI such as alectinib. The prospective phase II ASCEND-9 trial assessed the efficacy of ceritinib in patients with $A L K$-rearranged NSCLC who had disease progression on alectinib. This showed that the efficacy of ceritinib after alectinib was limited with an ORR of $25 \%$ (95\% CI, $8.7-49.1 \%$ ) and a DCR of $70 \%$ (95\% CI, $45.7-88.1 \%$ ) but with a median PFS of only 3.7 (95\% CI, 1.9-5.3) months (39). The use of brigatinib in alectinibrefractory $A L K$-rearranged NSCLC patients was also assessed and showed limited activity, with an ORR in $17 \%$ of patients with measurable disease and median PFS of 4.4 months in a retrospective study (21). Ensartinib was also assessed in this setting and showed an ORR of $23 \%$ and a DCR of $50 \%$ (24).

\section{Activity on various EML4-ALK variants}

Several EML4- $A L K$ variants have been previously reported. The most common are variant 1 (33\%), where exon 13 of EML4 is fused to exon 20 of $A L K$ (E13; A20). In variant 2 (10\%), exon 20 of EML4 is fused to exon 20 of $A L K$ (E20; $\mathrm{A} 20)$, and in variant $3 \mathrm{a} / \mathrm{b}(29 \%)$, exon $6 \mathrm{a}$ or $6 \mathrm{~b}$ of EML4 is fused to exon 20 of $A L K(\mathrm{E} 6 \mathrm{a} / \mathrm{b} ; \mathrm{A} 20)$ (40-42). In a Japanese study retrospectively evaluating the $A L K$ variants of 35 patients treated with crizotinib, the most common was variant 1 in 19 patients (54\%), followed by variant 2 in 5 patients (14\%), variant $3 \mathrm{a} / 3 \mathrm{~b}$ in 4 patients (12\%), and other variants in 7 patients $(20 \%)$.

The ORR was $69 \%$ in all patients, whereas it was $74 \%$ and $63 \%$ in the variant 1 and non-variant 1 groups, respectively. The median PFS time was significantly longer in patients with variant 1 than in those with non-variant 1 [median PFS, 11.0 (95\% CI, 6.5-43.0) vs. 4.2 (95\% CI, 1.610.2) months, respectively; $\mathrm{P}=0.05]$. Multivariable analysis determined two factors that were significantly associated with PFS duration which were having an $A L K$ variant 1 (HR, $0.350 ; 95 \% \mathrm{CI}, 0.128-0.929 ; \mathrm{P}=0.05)$ and advanced stage (HR, 4.646; 95\% CI, 1.381-21.750; $\mathrm{P}=0.05$ ) suggesting the 
better efficacy of crizotinib in patients with $A L K$ variant 1 versus non-variant 1 (43).

\section{IHC/FISH status}

$A L K$ rearrangements can be detected by IHC, FISH or NGS. However, whether to treat with an ALK TKI in cases of discordant IHC and FISH results remain a challenge. Data collected from the ALEX trial was further evaluated to examine clinical outcomes for IHC-positive/FISH-negative NSCLC patients treated with alectinib and crizotinib. IHC testing was performed using the VENTANA ALK (D5F3) CDx Assay and FISH testing was done with the Vysis ALK Break Apart FISH Probe Kit. In the ALEX trial, 303 patients with $A L K$ IHC positivity were randomized to receive either alectinib or crizotinib treatment. Two hundred and three patients also were found to $A L K$ FISH positive and 39 patients were found to be $A L K$ FISHnegative. Sixty-one of the $A L K$ IHC-positive patients did not have a corresponding FISH result due to uninformative FISH testing or inadequate tissue for testing. Exploratory analysis revealed that PFS of $A L K$ FISH-positive patients were similar to those of the IHC-positive patients. The $A L K$ IHC-positive/FISH-negative patients were found to derive clinical benefit from ALK TKI indicating that $A L K$ IHC testing may identify more patients who are eligible for ALK TKI (44).

\section{Intracranial activity}

Brain metastasis is relatively common in $A L K$-rearranged NSCLC patients and has been reported in approximately $20-30 \%$ of patients at initial diagnosis (45), and approximately $50 \%$ of patients will later develop CNS metastasis over the course of their disease (46). Crizotinib has limited CNS penetration due to a low CSF-to-plasma concentration ratio (0.0026) (47) which explains the reason why CNS is a common site of disease progression with crizotinib failure (45).

If the brain is the only site of progression, the use of later generation ALK inhibitors should be first considered (if not already utilized) and while radiation [stereotactic radiosurgery (SRS) or whole brain radiation (WBRT)] or surgical resection could be performed in select cases in those with large volume of disease and or those with symptomatic brain metastases. Typically, ALK TKIs are usually withheld on the days radiation therapy is being given and resumed after its completion to minimize the risk of potential neurotoxicity (48). It is important to note that the high CNS activity of alectinib, brigatinib and lorlatinib may allow clinicians to avoid the use of radiation in patients with asymptomatic CNS metastasis as there are reports of increased risk of radiation necrosis with concomitant use of ALK TKIs and cranial irradiation (49-51).

\section{Resistance mutations}

Initial responses to ALK inhibitors are not always durable, and eventually, virtually all patients develop tumor progression. Resistance can occur as on-target alterations such as $A L K$ gene mutations or amplifications, and offtarget events such as upregulation of bypass signaling pathways.

Molecular profiling at the time of diagnosis and also at the time of progression may assist in the sequential treatment selection of an ALK TKI, as specific $A L K$ resistance mutations may predict for sensitivity to certain ALK TKIs. While a positive liquid biopsy is often times sufficient to initiate targeted therapy (52), tissue biopsy should be encouraged in cases where liquid biopsy testing does not reveal the resistance mechanism and when rapid progression appears suspicious for small cell lung cancer transformation.

The most common secondary resistant $A L K$ mutant in patients post progression with second-generation ALK inhibitors is the Gly1202Arg (G1202R), which has been reported to occur in $21 \%$ of patients treated with ceritinib, $29 \%$ of patients on alectinib, and $43 \%$ of patients on brigatinib (25). The amino acid substitution of G1202R resides at the solvent-exposed region of ALK, where the bulkier, charged side chain is considered to cause steric interference with the binding of most ALK TKIs (25).

Specific $A L K$ fusion variants have demonstrated improved clinical outcome. For example, having the EML4$A L K$ variant 3 was significantly associated with developing $A L K$ resistance mutations, particularly at G1202R.

An exploratory analysis of 29 patients who received lorlatinib demonstrated that those with variant 3 had a significantly longer median PFS than those with variant 1 (11.0 vs. 3.3 months; $\mathrm{P}=0.011$ ). Therefore, this EML4-ALK variant 3 could represent a potential biomarker for response to lorlatinib (26).

The approval of lorlatinib was based on a phase II study with $215 A L K$-rearranged NSCLC patients who had failed at least one line of ALK inhibitors. Among patients who failed two or more ALK 
TKIs (including ceritinib and alectinib), ORR was $39 \%$ and median PFS was 6.9 months (9). In a subsequent analysis of tissue and plasma genotyping, those with mutations in the plasma $(62 \%)$ or tissue $(69 \%)$ showed higher responses to lorlatinib than those without mutations in the plasma (32\%) or tissue (7\%) (27), confirming the clinical utility of lorlatinib for on-target (ALK-dependent) resistance post early generation ALK inhibitors.

Lorlatinib has demonstrated efficacy in patients who were resistant to first and or second-generation ALK TKIs. However, acquired resistance to lorlatinib is unfortunately inevitable. Recently, the mechanisms of lorlatinib resistance have been characterized by sequencing lorlatinib-resistant biopsy samples from 20 patients. The dominant resistance mechanism to lorlatinib was primarily from multiple different compound $A L K$ mutations, or, a double mutation. For example, patients harboring $A L K \mathrm{C} 1156 \mathrm{Y}$ may develop resistance to lorlatinib by acquiring $A L K \mathrm{C} 1156 \mathrm{Y} /$ L1198F (53). Of interest, Shaw et al. has demonstrated that the double mutation of $A L K$ C1156Y/L1198F found at lorlatinib-resistance surprisingly restored its sensitivity to crizotinib, although the patient had previously failed crizotinib and ceritinib (54). This mimics the observation found in EGFR mutated patients who develop osimertinib third-generation EGFR TKI resistance through the C797S mutation, which has the potential to be overcome by gefitinib or erlotinib, both are first-generation EGFR TKIs.

In alectinib-refractory patients, brigatinib may represent a viable therapeutic option with those with tumors demonstrating I1171X or V1180L, but may not be as effective otherwise, including those with G1202R (21).

Molecular profiling may not need to be from tissue. Interestingly, a recent study reported by Horn et al., demonstrated the utility of circulating tumor DNA monitoring to analyze therapeutic response and resistance to ensartinib. In this study, patients with an EML4-ALK variant 1 (V1) fusion had improved response (9 of 17 patients; $53 \%)$ to ensartinib compared to patients with $E M L 4-A L K$ variant 3 (V3) fusion (1 of 7 patients; 14\%) (28).

\section{Toxicities}

While ALK inhibitors are generally better tolerated than chemotherapy, various toxicities have been reported. Vision disorder $(71 \%)$, diarrhea $(61 \%)$ and edema $(49 \%)$ were the most common adverse events reported with crizotinib use from the first-line study (10). In clinical practice, nausea and vomiting can often be attributed to crizotinib as well as ceritinib. The gastrointestinal toxicities of ceritinib were rather significant in the ASCEND-4 trial comparing ceritinib to chemotherapy. Indeed, dose decreases and interruption rates were higher in the ceritinib group (80\%) than in the chemotherapy arm (45\%) (11). Subsequent studies have shown better tolerance of ceritinib taken with food which is what is recommended on the current drug label (16). In the ALEX study, laboratory abnormalities such as anemia and liver enzyme alterations were the most commonly reported adverse events from alectinib (7). Clinically, alectinib has been associated with edema and myalgias whereas early-onset pulmonary toxicity has been reported with brigatinib. In the ALTA-1L trial, grade 3 or 4 pulmonary toxicity of interstitial lung disease or pneumonitis was reported in $3 \%$ of patients treated with brigatinib and $0.7 \%$ of those receiving crizotinib (8). All of the four events of interstitial lung disease or pneumonitis in the brigatinib arm were "early onset" as defined as manifesting within 14 days after the start of treatment and suggests the importance of close follow up during this critical time. Other adverse events reported with the use of brigatinib included diarrhea, nausea, increased blood creatinine kinase levels and increase in lipase levels.

The unique toxicity profile of lorlatinib is also something to note. Common adverse events include hyperlipidemia and many patients require the introduction or titration of statin therapy. Of note, pravastatin, rosuvastatin or pitavastatin should be initially considered due to their lower involvement with specific CYP450 enzymes that could interact with lorlatinib (55). Patients may also experience mild changes in mental status. Such effects reported in the phase I-II studies were generally mild and improved or resolved upon dose interruptions or reductions. There appears to be a wide range of CNS side effects and includes changes in cognitive function (i.e., memory impairment, confusion, disturbances in attention), mood (i.e., irritability, anxiety, depression, flat affect, euphoria/mania), and speech (i.e., slowed speech, difficulty in word finding). Out of 117 patients who had CNS effects, 24 (20.5\%) required one or more than one dose level of modification (such as temporary interruption and or dose reduction) with $15 / 24$ (62.5\%) of these patients having resolution of their CNS effects (56). The lorlatinib package insert recommends withholding the dose until grade 1 or lower and resuming at a reduced dose for grade 2 or 3 CNS effects and permanently discontinuing lorlatinib for grade 4 CNS events (57).

In general, NSCLC patients who harbor the $A L K$ 
Table 2 Trials evaluating combination of ALK TKIs and immune checkpoint inhibitors

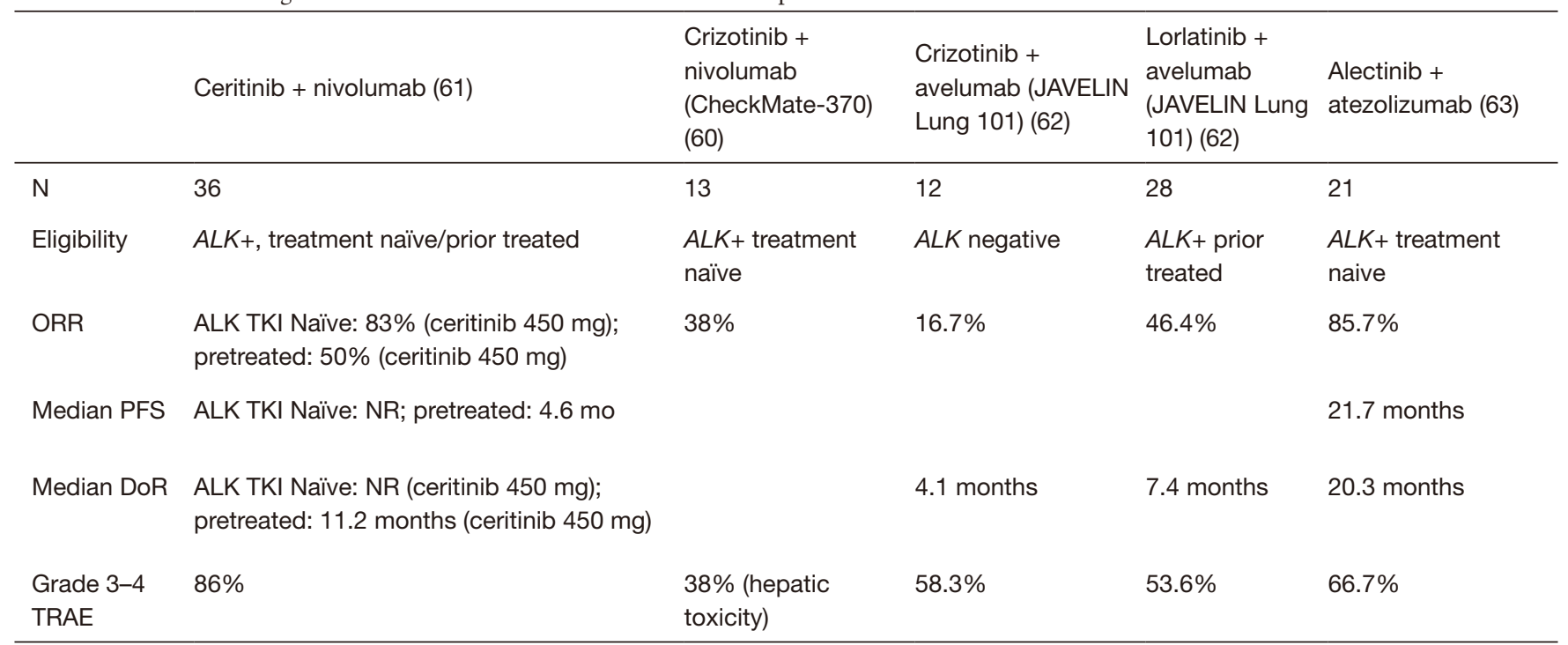

ALK, Anaplastic lymphoma kinase; TKIs, tyrosine kinase inhibitors; NR, not reached; ORR, overall response rate; PFS, progression free survival; DoR, duration of response; TRAE, treated related adverse events.

translocation are often young and highly functional. In those with occupations that require quick thinking and rapid processing (i.e., operation of machinery or stock exchange), lorlatinib may not be the optimal drug of choice. It is critical that not only patients but family members and care givers also be informed of the potential CNS effects of lorlatinib at the start of therapy and to be educated to notify their treating physicians as soon as such events were to occur.

\section{Ongoing studies, combo strategies}

Lorlatinib is in phase III testing to investigate whether firstline treatment with lorlatinib can further improve clinical outcomes for patients with metastatic $A L K$-rearranged NSCLC compared with first-line crizotinib treatment (ClinicalTrials.gov, NCT03052608, CROWN study). Given high CNS penetration of lorlatinib when compared to other ALK inhibitors, it would be important to document outcomes such as intracranial response rates and time to progression (which are included in the study's secondary objectives), in addition to the traditional primary outcome of PFS.

At the current time, there are no established treatment combination strategies that includes an ALK TKI. Important to note are the potential for developing $A L K$ nondominant mechanisms of resistance, which was actually documented in 12 out of $20(60 \%)$ in the aforementioned study by Yoda $e t$ al. Consideration of combination treatment with an ALK inhibitor with other agents that bypass different pathways such as MET, EGFR, KIT or SRC may be a valuable approach in those with $A L K$-nondominant mechanisms of resistance. For example, ALK inhibition with mitogen-activated protein kinase inhibition in mouse models have shown to prevent resistance and increase response to ALK inhibition (58) and this approach is being evaluated in clinical trials (NCT03202940: alectinib and cobimetinib, NCT03087448: ceritinib and trametinib).

Although immunotherapy has changed the landscape of NSCLC treatment, there are data to show that using single agent immune checkpoint inhibitors in those patients with sensitizing $A L K$-rearrangements may not be as efficacious (59) with the potential for increased toxicity when combined with an ALK TKI; indeed, enrollment for CheckMate-370, a study combining crizotinib and nivolumab as first-line treatment was discontinued due to significant hepatotoxicity (60). Therefore, careful selection of such combination or sequential approaches with immunotherapy must be examined.

Trials evaluating combination of ALK TKI and immune checkpoint inhibitors is shown in Table 2 (60-63).

The ALK Master Protocol/NRG Intergroup trial (NCT03737994) is an important study. In this trial, patients 
who have progressed on any ALK TKI will undergo a biopsy and a blood-based plasma test to look for acquired resistance mechanisms. Based on the results, the patients are assigned to an arm of different ALK inhibitors with or without combination of other targeted therapies. For example, those found to have $A L K 1198 \mathrm{~F}$ mutation or MET amplification will be assigned to receive crizotinib whereas those with compound mutations receive lorlatinib. Those with no $A L K$-resistance mutations are assigned to either lorlatinib, ceritinib, alectinib, brigatinib, ensartinib or pemetrexed with or without cisplatin/carboplatin.

In the adjuvant setting, the phase III ALCHEMIST screening trial (NCT02194738) is evaluating the utility of ALK TKIs in patients with early-stage $A L K$ rearranged NSCLC who have completed standard treatment such as chemotherapy after surgical resection. For the neoadjuvant setting, the Lung Cancer Mutation Consortium has proposed the PROMISE umbrella trial (LCMC4) to test for the presence of oncogenic drivers at diagnosis in patients with early stage lung cancer and to provide matched targeted therapies prior to surgical resection (64). Although there are currently five approved ALK inhibitors for NSCLC, there are currently no tumor agnostic approvals at this time. The My Pathway basket trial (NCT02091141) has a cohort evaluating the activity of alectinib in patients with ALK-mutated tumors (that are not NSCLC such as neuroblastoma and inflammatory myofibroblastic tumors).

\section{Cost effectiveness}

Studies evaluating the cost effectiveness of ALK TKIs are limited but generally supports the idea of utilizing secondgeneration ALK TKIs upfront. A US based study has shown that ceritinib is cost-effective when compared to chemotherapy and crizotinib in the treatment of treatment naïve ALK-positive metastatic NCSLC. Zhou et al., reported that upfront use of ceritinib was associated with total direct costs of $\$ 299,777$ and 3.28 QALYs [from 4.61 life years gained (LYG)] over 20 years whereas upfront use of crizotinib and chemotherapy were associated with 2.73 and 2.41 QALYs, 3.92 and 3.53 LYG, and \$263,172 and $\$ 228,184$ total direct costs, respectively. The incremental cost per QALY gained was $\$ 66,064$ for ceritinib vs. crizotinib and $\$ 81,645$ for ceritinib vs. chemotherapy. In the first 2 years after starting treatment, ceritinib dominated crizotinib by conferring greater health benefits at reduced total costs (65).
Prevention of brain metastases utilizing alectinib may also be cost-effective. Considering the incidence of brain metastases in each treatment arm of the ALEX trial and costs associated with brain metastases, Burudpakdee et al., estimated that over the 24-month follow-up period, treatment with alectinib rather than crizotinib, could save on average between $\$ 35,254$ and $\$ 41,434$ per patient in healthcare costs related to brain metastases (66). As $45.3 \%$ of patients treated with crizotinib developed intracranial metastases compared to $7.2 \%$ of patients treated with alectinib, the potential cost savings of preventing brain metastases in ALK+ NSCLC patients appears to be significant.

\section{Conclusions}

ALK inhibitors have robust efficacy in $A L K$-rearranged lung cancers. Second-generation ALK inhibitors, alectinib and brigatinib have demonstrated superior PFS and CNS activity compared to first-generation TKI, crizotinib, in treatment naïve patients and generally used initially in the first-line setting. However, responses are not always durable and a majority of patients will develop resistance. Molecular sequencing at time of resistance may assist in the sequential selection of an ALK inhibitor. The development of $A L K$ resistance mutations, particularly $\mathrm{G} 1202 \mathrm{R}$ was associated significantly with having an $E M L 4-A L K$ variant 3. Lorlatinib has demonstrated efficacy in $E M L 4-A L K$ variant 3 and has been shown to be effective after resistance to first and second-generation ALK TKIs. Additional research to evaluate mechanisms of acquired resistance will be crucial to the development of next generation ALK TKIs.

\section{Acknowledgments}

Funding: None.

\section{Footnote}

Provenance and Peer Review: This article was commissioned by the Guest Editors (Silvia Novello, Francesco Passiglia) for the series "Looking for Chimeras in NSCLC: Widen Therapeutic Options Targeting Oncogenic Fusions" published in Translational Lung Cancer Research. The article was sent for external peer review organized by the Guest Editors and the editorial office.

Peer Review File: Available at http://dx.doi.org/10.21037/ 
tlcr-20-331

Conflicts of Interest: All authors have completed the ICMJE uniform disclosure form (available at http:// dx.doi.org/10.21037/tlcr-20-331). The series "Looking for Chimeras in NSCLC: Widen Therapeutic Options Targeting Oncogenic Fusions" was commissioned by the editorial office without any funding or sponsorship. SHIO serves as an unpaid editorial board member of Translational Lung Cancer Research from Jun 2018 to Jun 2020. BX has received honorarium from Astra Zeneca and advisory fees from Astra Zeneca, Roche/Genentech, and Regeneron Pharmaceuticals, outside the submitted work. $\mathrm{MN}$ has received advisory fees from AstraZeneca, Caris Life Sciences, Daiichi Sankyo, Takeda, Novartis, research support from Tempus and travel support from An Heart Therapeutics, outside the submitted work. VWZ has received honoraria from AstraZeneca, Biocept, Roche-Foundation Medicine, Roche/Genentech, Takeda, and has stock ownership in TP Therapeutics, outside the submitted work. SHIO has stock ownership and was on the scientific advisory board of Turning Point Therapeutics Inc (until Feb 28, 2019), and has received speaker honorarium from Merck, Roche/Genentech, Astra Zeneca, Takeda/ARIAD and Pfizer; has received advisory fees from Roche/Genentech, Astra Zeneca, Takeda/ARIAD, Pfizer, Foundation Medicine Inc, Spectrum, outside the submitted work. RAS has received honoraria from AstraZeneca, AMGEN, BMS, Boehringer Ingelheim, Lilly, Merck, Novartis, Pfizer, Roche, Taiho, Takeda, and Yuhan; and research funding from Astra-Zeneca and Boehringer Ingelheim, outside the submitted work. The authors have no other conflicts of interest to declare.

Ethical Statement: The authors are accountable for all aspects of the work in ensuring that questions related to the accuracy or integrity of any part of the work are appropriately investigated and resolved.

Open Access Statement: This is an Open Access article distributed in accordance with the Creative Commons Attribution-NonCommercial-NoDerivs 4.0 International License (CC BY-NC-ND 4.0), which permits the noncommercial replication and distribution of the article with the strict proviso that no changes or edits are made and the original work is properly cited (including links to both the formal publication through the relevant DOI and the license).
See: https://creativecommons.org/licenses/by-nc-nd/4.0/.

\section{References}

1. Bray F, Ferlay J, Soerjomataram I, et al. Global cancer statistics 2018: GLOBOCAN estimates of incidence and mortality worldwide for 36 cancers in 185 countries. CA Cancer J Clin 2018;68:394-424.

2. Shaw AT, Solomon B. Targeting anaplastic lymphoma kinase in lung cancer. Clin Cancer Res 2011;17:2081-6.

3. Pikor LA, Ramnarine VR, Lam S, et al. Genetic alterations defining NSCLC subtypes and their therapeutic implications. Lung Cancer 2013;82:179-89.

4. Shaw AT, Yeap BY, Mino-Kenudson M, et al. Clinical features and outcome of patients with non-small-cell lung cancer who harbor EML4-ALK. J Clin Oncol 2009;27:4247-53.

5. Ou SHI, Zhu VW, Nagasaka M. Catalog of 5' Fusion Partners in ALK-positive NSCLC Circa 2020. JTO Clinical and Research Reports 2020;1:100015.

6. Shaw AT, Kim DW, Mehra R, et al. Ceritinib in ALKrearranged non-small-cell lung cancer. $\mathrm{N}$ Engl J Med 2014;370:1189-97.

7. Peters S, Camidge DR, Shaw AT, et al. Alectinib versus crizotinib in untreated ALK-positive non-small-cell lung cancer. N Engl J Med 2017;377:829-38.

8. Camidge DR, Kim HR, Ahn MJ, et al. Brigatinib versus crizotinib in ALK-positive non-small-cell lung cancer. N Engl J Med 2018;379:2027-39.

9. Solomon BJ, Besse B, Bauer TM, et al. Lorlatinib in patients with ALK-positive non-small-cell lung cancer: results from a global phase 2 study. Lancet Oncol 2018;19:1654-67.

10. Solomon BJ, Mok T, Kim DW, et al. First-line crizotinib versus chemotherapy in ALK-positive lung cancer. $\mathrm{N}$ Engl J Med 2014;371:2167-77.

11. Soria JC, Tan DSW, Chiari R, et al. First-line ceritinib versus platinum-based chemotherapy in advanced ALKrearranged non-small-cell lung cancer (ASCEND-4): a randomised, open-label, phase 3 study. Lancet 2017;389:917-29.

12. Camidge DR, Dziadziuszko R, Peters S, et al. Updated efficacy and safety data and impact of the EML4-ALK fusion variant on the efficacy of alectinib in untreated ALK-positive advanced non-small cell lung cancer in the global phase III ALEX study. J Thorac Oncol 2019;14:1233-43. 
13. Camidge R, Kim HR, Ahn M, et al. Brigatinib vs crizotinib in patients with ALK inhibitor-naive advanced ALK+ NSCLC: Updated results from the phase III ALTA1L trial. Ann Oncol 2019;30:ix183-202.

14. Horn L, Infante JR, Reckamp KL, et al. Ensartinib (X-396) in ALK-positive non-small cell lung cancer: results from a first-in-human phase I/II, multicenter study. Clin Cancer Res 2018;24:2771-9.

15. Mok TS, Kim D, Wu Y, et al. Overall survival (OS) for first-line crizotinib versus chemotherapy in ALK+ lung cancer: updated results from PROFILE 1014. Ann Oncol 2017;28:v605-49.

16. Cho BC, Kim DW, Bearz A, et al. ASCEND-8: A randomized phase 1 study of ceritinib, $450 \mathrm{mg}$ or 600 $\mathrm{mg}$, taken with a low-fat meal versus $750 \mathrm{mg}$ in fasted state in patients with anaplastic lymphoma kinase (ALK)-rearranged metastatic non-small cell lung cancer (NSCLC). J Thorac Oncol 2017;12:1357-67.

17. Peters S, Mok T, Gadgeel SM, et al. Updated overall survival (OS) and safety data from the randomized, phase III ALEX study of alectinib (ALC) versus crizotinib (CRZ) in untreated advanced ALK+ NSCLC. J Clin Oncol 2020;38:abstr 9518.

18. Hida T, Nokihara H, Kondo M, et al. Alectinib versus crizotinib in patients with ALK-positive non-small-cell lung cancer (J-ALEX): an open-label, randomised phase 3 trial. Lancet 2017;390:29-39.

19. Zhou C, Kim SW, Reungwetwattana T, et al. Alectinib versus crizotinib in untreated Asian patients with anaplastic lymphoma kinase-positive non-small-cell lung cancer (ALESIA): a randomised phase 3 study. Lancet Respir Med 2019;7:437-46.

20. Gadgeel SM, Mok T, Peters S, et al. Phase II/III Blood First Assay Screening Trial (BFAST) in patients (pts) with treatment-naïve NSCLC: initial results from the ALK+ cohort. Ann Oncol 2019;30:v851-934.

21. Lin JJ, Zhu VW, Schoenfeld AJ, et al. Brigatinib in patients with alectinib-refractory ALK-positive NSCLC.J Thorac Oncol 2018;13:1530-8.

22. Shaw AT, Felip E, Bauer TM, et al. Lorlatinib in nonsmall-cell lung cancer with ALK or ROS1 rearrangement: an international, multicentre, open-label, single-arm firstin-man phase 1 trial. Lancet Oncol 2017;18:1590-9.

23. Syed YY. Lorlatinib: first global approval. Drugs 2019;79:93-8.

24. Horn L, Leal TA, Oxnard G, et al. OA03.08 Activity of ensartinib after second generation anaplastic lymphoma kinase (ALK) tyrosine kinase inhibitors (TKI): topic: medical oncology. J Thorac Oncol 2017;12:S1556.

25. Gainor JF, Dardaei L, Yoda S, et al. Molecular mechanisms of resistance to first- and second-generation ALK inhibitors in ALK-rearranged lung cancer. Cancer Discov 2016;6:1118-33.

26. Lin JJ, Zhu VW, Yoda S, et al. Impact of EML4-ALK variant on resistance mechanisms and clinical outcomes in ALK-positive lung cancer. J Clin Oncol 2018;36:1199-206.

27. Shaw AT, Solomon BJ, Besse B, et al. ALK resistance mutations and efficacy of lorlatinib in advanced anaplastic lymphoma kinase-positive non-small-cell lung cancer. J Clin Oncol 2019;37:1370-9.

28. Horn L, Whisenant JG, Wakelee H, et al. Monitoring therapeutic response and resistance: analysis of circulating tumor DNA in patients with ALK+ lung cancer. J Thorac Oncol 2019;14:1901-11.

29. Gadgeel S, Peters S, Mok T, et al. Alectinib versus crizotinib in treatment-naive anaplastic lymphoma kinase-positive $(\mathrm{ALK}+)$ non-small-cell lung cancer: CNS efficacy results from the ALEX study. Ann Oncol 2018;29:2214-22.

30. Califano R, Hochmair MJ, Gridelli C, et al. Brigatinib (BRG) vs crizotinib (CRZ) in the phase 3 ALTA-1L trial. Ann Oncol 2019;30:ii38-68.

31. ESMO. Clinical Practice Living Guidelines - Metastatic Non-Small-Cell Lung Cancer. Available online: https:// www.esmo.org/guidelines/lung-and-chest-tumours/ metastatic-non-small-cell-lung-cancer (Accessed 1 March, 2020).

32. Duruisseaux M, Besse B, Cadranel J, et al. Overall survival with crizotinib and next-generation ALK inhibitors in ALK-positive non-small-cell lung cancer (IFCT-1302 CLINALK): a French nationwide cohort retrospective study. Oncotarget 2017;8:21903-17.

33. Gainor JF, Tan DS, De Pas T, et al. Progression-free and overall survival in alk-positive NSCLC patients treated with sequential crizotinib and ceritinib. Clin Cancer Res 2015;21:2745-52.

34. Chiari R, Metro G, Iacono D, et al. Clinical impact of sequential treatment with ALK-TKIs in patients with advanced ALK-positive non-small cell lung cancer: results of a multicenter analysis. Lung Cancer 2015;90:255-60.

35. Ito K, Hataji O, Kobayashi H, et al. Sequential therapy with crizotinib and alectinib in ALK-rearranged nonsmall cell lung cancer-a multicenter retrospective study. J Thorac Oncol 2017;12:390-6.

36. Iams WT, Lovly CM. Anaplastic lymphoma kinase as a therapeutic target in non-small cell lung cancer. Cancer J 
2015;21:378-82.

37. NCCN. Guidelines for Non-Small Cell Lung Cancer. Available online: https://www.nccn.org/professionals/ physician_gls/pdf/nscl.pdf (Accessed 1 March, 2020).

38. Lin JJ, Schoenfeld AJ, Zhu VW, et al. Efficacy of platinum/ pemetrexed combination chemotherapy in ALK-positive NSCLC refractory to second-generation ALK inhibitors. J Thorac Oncol 2020;15:258-65.

39. Hida T, Seto T, Horinouchi H, et al. Phase II study of ceritinib in alectinib-pretreated patients with anaplastic lymphoma kinase-rearranged metastatic non-smallcell lung cancer in Japan: ASCEND-9. Cancer Sci 2018;109:2863-72.

40. Choi YL, Soda M, Yamashita Y, et al. EML4-ALK mutations in lung cancer that confer resistance to ALK inhibitors. N Engl J Med 2010;363:1734-9.

41. Sasaki T, Rodig SJ, Chirieac LR, et al. The biology and treatment of EML4-ALK non-small cell lung cancer. Eur J Cancer 2010;46:1773-80.

42. Horn L, Pao W. EML4-ALK: honing in on a new target in non-small-cell lung cancer. J Clin Oncol 2009;27:4232-5.

43. Yoshida T, Oya Y, Tanaka K, et al. Differential crizotinib response duration among ALK fusion variants in ALKpositive non-small-cell lung cancer. J Clin Oncol 2016;34:3383-9.

44. Mok T, Peters S, Camidge DR, et al. MA 07.01 Patients with ALK IHC-positive/FISH-negative NSCLC benefit from ALK TKI treatment: response data from the global ALEX trial. J Thorac Oncol 2017;12:S1826.

45. Costa DB, Shaw AT, Ou SH, et al. Clinical experience with crizotinib in patients with advanced ALK-rearranged non-small-cell lung cancer and brain metastases. J Clin Oncol 2015;33:1881-8.

46. Rangachari D, Yamaguchi N, VanderLaan PA, et al. Brain metastases in patients with EGFR-mutated or ALKrearranged non-small-cell lung cancers. Lung Cancer 2015;88:108-11.

47. Costa DB, Kobayashi S, Pandya SS, et al. CSF concentration of the anaplastic lymphoma kinase inhibitor crizotinib. J Clin Oncol 2011;29:e443-5.

48. Weickhardt AJ, Scheier B, Burke JM, et al. Local ablative therapy of oligoprogressive disease prolongs disease control by tyrosine kinase inhibitors in oncogeneaddicted non-small-cell lung cancer. J Thorac Oncol 2012;7:1807-14.

49. Ou SH, Weitz M, Jalas JR, et al. Alectinib induced CNS radiation necrosis in an ALK+NSCLC patient with a remote ( 7 years) history of brain radiation. Lung Cancer 2016;96:15-8.

50. Ou SH, Klempner SJ, Azada MC, et al. Radiation necrosis presenting as pseudoprogression (PsP) during alectinib treatment of previously radiated brain metastases in ALKpositive NSCLC: implications for disease assessment and management. Lung Cancer 2015;88:355-9.

51. Zhu VW, Nagasaka M, Kubota T, et al. Symptomatic CNS radiation necrosis requiring neurosurgical resection during treatment with lorlatinib in ALK-rearranged NSCLC: a report of two cases. Lung Cancer (Auckl) 2020;11:13-8.

52. Rolfo C, Mack PC, Scagliotti GV, et al. Liquid biopsy for advanced non-small cell lung cancer (NSCLC): a statement paper from the IASLC. J Thorac Oncol 2018;13:1248-68.

53. Yoda S, Lin JJ, Lawrence MS, et al. Sequential ALK inhibitors can select for lorlatinib-resistant compound ALK mutations in ALK-positive lung cancer. Cancer Discov 2018;8:714-29.

54. Shaw AT, Friboulet L, Leshchiner I, et al. Resensitization to crizotinib by the lorlatinib ALK resistance mutation L1198F. N Engl J Med 2016;374:54-61.

55. Neuvonen PJ, Niemi M, Backman JT. Drug interactions with lipid-lowering drugs: mechanisms and clinical relevance. Clin Pharmacol Ther 2006;80:565-81.

56. Bauer TM, Felip E, Solomon BJ, et al. Clinical management of adverse events associated with lorlatinib. Oncologist 2019;24:1103-10.

57. Highlights of prescribing information: Lorbrena (lorlatinib). Available online: https:/www.accessdata. fda.gov/drugsatfda_docs/label/2018/210868s000lbl.pdf (Accessed 1 April, 2020).

58. Hrustanovic G, Olivas V, Pazarentzos E, et al. RASMAPK dependence underlies a rational polytherapy strategy in EML4-ALK-positive lung cancer. Nat Med 2015;21:1038-47.

59. Gainor JF, Shaw AT, Sequist LV, et al. EGFR mutations and ALK rearrangements are associated with low response rates to $\mathrm{PD}-1$ pathway blockade in non-small cell lung cancer: a retrospective analysis. Clin Cancer Res 2016;22:4585-93.

60. Spigel DR, Reynolds C, Waterhouse D, et al. Phase $1 / 2$ study of the safety and tolerability of nivolumab plus crizotinib for the first-line treatment of anaplastic lymphoma kinase translocation - positive advanced nonsmall cell lung cancer (CheckMate 370). J Thorac Oncol 2018;13:682-8.

61. Felip E, de Braud FG, Maur M, et al. Ceritinib plus 
nivolumab in patients with advanced ALK-rearranged non-small cell lung cancer: results of an openlabel, multicenter, phase 1B study. J Thorac Oncol 2020;15:392-403.

62. Shaw AT, Lee S-H, Ramalingam SS, et al. Avelumab (antiPD-L1) in combination with crizotinib or lorlatinib in patients with previously treated advanced NSCLC: phase $1 \mathrm{~b}$ results from JAVELIN Lung 101. J Thorac Oncol 2018;36:9008.

63. Kim D-W, Gadgeel SM, Gettinger SN, et al. Safety and clinical activity results from a phase Ib study of alectinib plus atezolizumab in ALK+ advanced NSCLC (aNSCLC). J Thorac Oncol 2018;36:9009.

Cite this article as: $\mathrm{Xia} \mathrm{B}$, Nagasaka $\mathrm{M}$, Zhu VW, Ou SHI, Soo RA. How to select the best upfront therapy for metastatic disease? Focus on $A L K$-rearranged non-small cell lung cancer (NSCLC). Transl Lung Cancer Res 2020;9(6):2521-2534. doi: 10.21037/tlcr-20-331
64. Bauer TM, Papadopoulos KP, Autio KA, et al. A firstin-human study of pegylated recombinant human IL10 (AM0010), daily administered for four months in selected advanced solid tumors. ASCO Meeting Abstracts 2014;32:TPS3126.

65. Zhou ZY, Mutebi A, Han S, et al. Cost-effectiveness of ceritinib in previously untreated anaplastic lymphoma kinase-positive metastatic non-small cell lung cancer in the United States. J Med Econ 2018;21:577-86.

66. Burudpakdee C, Wong W, Seetasith A, et al. Economic impact of preventing brain metastases with alectinib in ALK-positive non-small cell lung cancer. Lung Cancer 2018;119:103-11. 